\title{
Déconstruction et renouveau esthétique: une exégèse narratologique de l'hybride et de la traduction dans Les Soleils des indépendances et Solibo Magnifique
}

\author{
Mouhamédoul A Niang \\ Colby College
}

\section{Introduction}

Les Soleils des indépendances et Solibo Magnifique traitent de la mort, de l'impact de la modernité sur le culturel, des rapports entre le français et une langue locale, et de la disparition imminente d'une voix ou d'une lignée. Ils illustrent une nouvelle pratique littéraire investie dans le dialogue linguistique et la déconstruction de la hiérarchisation des langues. Toute étude narratologique du premier roman devrait nous faire voir que la narration y est à la fois un jeu ou un exercice d'hybridation de la langue française et une exposition de la culture malinké devant un public cible francophone de prime abord. Ce jeu se fait par des voies structurelles, syncrétiques et sémantiques particulières; il s'effectue aussi à travers une mise en rapport du langage et de la littérature selon la logique de Roland Barthes. En effet, pour cet auteur, « [...] il n'est guère plus possible de concevoir la littérature comme un art qui se désintéresserait de tout rapport avec le langage... » (4). Ainsi, la narrativité $^{1}$ de ce texte romanesque révèle un entrecroisement de la langue française et du fait culturel malinké empreint d'oralité. Cependant, cette nouvelle langue hybride qui provient d'une traduction de certains aspects de la culture et de l'existence malinké en français dans Les Soleils des indépendances, même si elle est comprise ${ }^{2}$, ne reflète pas le vécu langagier des Malinkés. Il en est autrement dans Solibo Magnifique où l'hybridité linguistique transcende le romanesque en vertu de sa réalité socioculturelle. Les structures du récit de ce roman sont configurées selon le modèle de la poétique du divers kaléidoscopique préconisée dans Éloge de la Créolité (1989). Chez Kourouma, cette hybridité s'inscrit dans un acte d'écriture qui tend à démystifier le français en se l'appropriant par le biais d'une traduction linguistique et sémantique qui approxime deux univers symboliques.

Par traduction, nous entendons tout jeu d'équivalences ${ }^{3}$ entre deux champs sémantiques et culturels passant par un comparatif, une métaphore ou par une pratique de la paraphrase tendant à rendre intelligible un énoncé culturel opaque dans son agencement liminaire. Ce dernier cas de figure transparaît particulièrement dans le roman de Chamoiseau. Quant à l'œuvre de Kourouma, nous notons que sa pratique de la traduction comme moyen de rapprochement culturel ou

\footnotetext{
${ }_{1}^{1}$ Selon Mieke Bal, «La narrativité d'un texte est la manière dont le texte se laisse décoder comme narratif. Ainsi on peut dire que la narrativité est déterminée par les relations entre le texte narratif, le récit et l'histoire. D’où cette précision : la narratologie est la science qui cherche à formuler la théorie des relations entre texte narratif, récit et histoire. Elle ne s'occupera ni du texte narratif, ni de l'histoire pris isolément» (5).

${ }^{2}$ Kourouma atteste de ce fait comme suit: «Des gens sont condamnés à des ans, cinq ans, six ans pour un mot qui ne peut pas être traduit dans leur langue nationale. Il y a là quelque chose. Si vous voulez, moi-même étant malinké, j'en utilise, mais ce sont des mots qui sont compris. Par exemple, on a eu à discuter ce matin la phrase qui dit: "Il y avait une semaine qu'avait fini dans la capitale Koné Ibrahima." Tous les Africains comprennent cela. Les Mossis, [...] tout le monde. Je crois que c'est la cosmogonie africaine que je voudrais introduire dans le français pour que nous nous sentions chez nous en français » (Ouedraogo 774).

${ }^{3}$ Notre définition de la traduction prend en compte l'objectif que lui donne Pratt, à savoir « [t]ranslation in its normative, linguistic sense seeks some form of equivalence » (33). Dans Les Soleils des indépendances, cette equivalence introduite par le comparatif "comme"a une valeur sémantique et culturelle.
} 
d'intercompréhension se situe dans une longue tradition de traduction en terre africaine. Nous retenons à cet égard l'argument de Paul Bandia selon qui « [i]t is safe to assume that, given the plethora of languages and the great many ethnic groups on the African continent there must have been a great deal of translation and intercultural activity among these different peoples » (210). Le rôle dévolu à l'interprète africain pendant la période coloniale témoigne de sa prégnance en Afrique ${ }^{4}$. Chez Kourouma toujours, la pratique de la traduction a des affinités avec la stratégie de transculturation que Mary Louise Pratt théorise comme «[...] a 'phenomenon of the contact zone', a process where 'subordinated or marginalized groups select or invent from materials transmitted to them by a dominant or metropolitan culture', determining 'to varying extents what they absorb into their own, and 'what they use it or' » (Parry 8-9).

Nous proposons une lecture qui fait de la traduction romanesque en périphérie un pilier incontournable de l'hybridité dans ces œuvres. Par hybridité, nous entendons, à l'instar de Homi Bhabha, Stuart Hall et Françoise Lionnet, «the triumph of the postcolonial or the subaltern over the hegemonic » (Prabhu 12). Notre façon d'aborder cet entre-deux renvoie ici à la fois au concept bakhtinien de l'hybride romanesque ${ }^{5}$ et au rejet de l'écriture du registre par Chamoiseau et Confiant. Selon ces créolistes, "[l]'écriture du registre est prédatrice et aveugle. Simplifiante, elle précipite le Divers dans la pensée de l'Un; le grouillement, momifié dans le Même. L'écriture du registre espagnol ou français ignore la créolisation et ne témoigne que d'une aventure solitaire et hautaine » (27). Ainsi, les romans de Kourouma et Chamoiseau comportent diverses composantes narratologiques résistant à un paradigme esthétique ou linguistique rigide et unitaire. Ils mettent en branle une esthétique de la traduction qui n'est pas à envisager en termes de complexe d'infériorité culturelle. ${ }^{6}$ Elle recèle des caractéristiques relevant à la fois de la "visée éthique positive de la traduction » et de l'approche inscrite dans la « voie du centre »(Bandia 126, 133). S'appuyant sur certaines analyses de Berman, entre autres théoriciens, Bandia identifie une «visée éthique positive de la traduction» en termes d'《étrangeté», de " décentrement » et d' " ouverture sur l'Autre » (126). A celle-ci vient s'ajouter celle préconisée par Venuti, à savoir la traduction comme la "mise en œuvre de deux stratégies » débouchant sur " une troisième voie, la voie du centre (textual middles), caractérisée par des degrés variables de pratique cibliste et sourcière » (Bandia 132). Les Soleils des indépendances et Solibo Magnifique comportent des structures narratives qui laissent entrevoir une pratique de la traduction à la fois comme ouverture à l'autre et comme troisième espace.

\section{L'Analogie au service d'un renouvellement esthétique et d'un dire interculturel}

Le tissu narratif de Les Soleils des indépendances renferme un mélange de stratégies discursives, didactiques et descriptives. En tant que «fonction ${ }^{7}$, la première phrase du roman énonce la composante tripartite de cette stratégie narratologique amalgamée et protéiforme: « Il y avait une semaine qu'avait fini dans la capitale Koné Ibrahima, de race malinké, ou disons-le en malinké: il

\footnotetext{
${ }^{4}$ Voir Amadou Hampâté Bâ, Oui mon commandant! Paris, Actes Sud, 1996.

${ }^{5}$ Robert Young reprend ainsi cette définition traduite: "[T] he novelistic hybrid is an artistically organized system for bringing different languages in contact with one another, a system having as its goal the illumination of one language by means of another, the carving-out of a living image of another language. » (361 dans le texte original) (22).

${ }^{6}$ Cette pratique ne se retrouve guère dans l'analyse de Weinberger pour qui « [o]ne of the great spurs to translation is a cultural inferiority complex or a national self-loathing » (Pratt 29). Kourouma et Chamoiseau s'identifient fièrement à leurs héritages linguistiques respectifs.

${ }^{7}$ Parlant des trois niveaux du récit, Barthes note que « [1]'âme de toute fonction, c'est, si l'on peut dire, son germe, ce qui lui permet d'ensemencer le récit d'un élément qui mûrira plus tard, sur le même niveau, ou ailleurs, sur un autre niveau... »(7).
} 
n'avait pas soutenu un petit rhume » (Soleils 9). Dans ce récit annonciateur du deuil où une voix impersonnelle est relayée par une voix collective marque aussi l'appropriation, en toute indépendance, d'une langue et d'une forme littéraire héritées de la colonisation, le français et le roman en l'occurrence, à des fins d'hybridation textuelle et de traduction culturelle. L'« unité narrative » déjà énoncée introduit une méthode de traduction qui tantôt invente un nouveau sens au lexique français ${ }^{8}$ en l'imprégnant d'un autre réel, tantôt établit un rapport analogique ou crée un effet de miroir entre deux Weltanschanung ou vécus sociolinguistiques distincts. Kourouma y adopte la " visée éthique de la traduction positive » en dépouillant son texte de tout purisme linguistique ethnocentrique. Mieux, tout porte à croire qu'il la situe dans une esthétique de troisième espace par sa façon de faire paraitre certaines « caractéristiques de la [...] culture source », le malinké, à travers le français et le roman ou " espace littéraire de la culture réceptrice » (Bandia 133). Tout cela témoigne alors d'une corrélation entre l'hybridité et la traduction, la première s'aidant de la seconde dans Les Soleils des indépendances. On note en effet chez Kourouma une tendance à traduire presque systématiquement l'imaginaire malinké par une langue française dépaysée, décentrée et appropriée par la périphérie. Cette posture esthétique et relationnelle ${ }^{9}$ relève de la poétique de transculturation puisqu'elle est l'aboutissement d'un désir d'alléger les lourdeurs de la langue française afin qu'elle se prête plus facilement à l'expression d'un imaginaire africain jadis victime d'une violence épistémique. ${ }^{10}$ L'aveu de Kourouma ne laisse planer aucun doute d'ailleurs: «J'ai [...] traduit le malinké en français en cassant le français pour trouver et restituer le rythme africain » (Moncef 7). C'est par rapport à cette posture que nous proposons une lecture du vocable "comme", un comparatif qui illustre l'inscription du concept d'hybride romanesque dans la traduction chez Kourouma.

Aucune des plus récentes études portant sur la langue et la traduction dans Les Soleils des indépendances n'a souligné l'importance de ce terme analogique pour la narrativité esthétique de la traduction chez Kourouma. ${ }^{11}$ L'auteur emploie pourtant à foison une technique narrative qui consiste à relier deux références culturelles étrangères par le comparatif "comme", l'une renforçant ou éclairant l'autre dans un style à la fois répétitif et comique. Le "comme" relève ici de la glose et d'une clarification parfois redondante. Il traduit aussi le caractère aberrant de la suffisance d'une langue ou d'une référentialité culturelle autonome. Par l'usage du "comme", Kourouma parvient à battre en brèche l'unilatéralisme sociolinguistique dont la mission civilisatrice s'était fait le chantre. À l'instar de Cheikh Anta Diop qui recourut à la traduction pour faire participer la langue wolof au transfert et à l'expression de la science ${ }^{12}$, Kourouma fait expressément entrer l'imaginaire malinké

8 Voir, par exemple, Makhily Gassama, La Langue d'Abmadou Kourouma ou le français sous le soleil d'Afrique, Paris, Karthala, 1995.

9 Voir la lecture qu'en fait David Caron dans son article "Pour une poétique de l'opacité : Ahmadou Kourouma, Edouard Glissant et l'espace de la Relation francophone », Revue canadienne de littérature comparée 25.3-4 (1998): 348-362. Caron note, par exemple, que l'usage de la langue française par les auteurs africains comme Kourouma « appelle à la Relation, et pour peu qu'on l'assume, impose cette irruption du Divers dont parle Glissant » (350).

${ }^{10}$ Celia Britton reprend ainsi l'argument de Gayatri Spivak sur la violence épistémique: « She [Spivak] argues that it not only characterizes the imperialist project but also continues to operate in postcolonial societies to exclude and silence subaltern groups, that is those outside the new bourgeois nationalist ruling class » (20).

11 Voir entre autres analyses, Amadou Koné, « Discourse in Kourouma’s novels: Writing Two Languages to Translate Two Languages, » Research in African literatures 38.2 (2007), 109-123 et Kwaku A. Gyasi, "The African Writer as Translator: Writing African Languages through French Author(s), » Journal of African Cultural Studies 16.2 (2003), 143-159.

12 Bandia inscrit la traduction en wolof de la Théorie de la Relativité d'Albert Einstein par Cheikh Anta Diop dans l'effort du chercheur sénégalais de déconstruire les hypothèses pseudo-scientifiques à l'encontre des langues africaines, celles-ci étant acculées du stigma de non-scientificité. Il s'agit ici aussi d'une traduction au service de la reconstruction de l'histoire (212). 
dans la modernité du texte romanesque par ce même processus. La trame narrative de Les Soleils des indépendances regorge de passages où le "comme" participe à cet acte historique. Notons que ce roman a été affublé du titre de "premier roman moderne”. C'est à travers le récit des vicissitudes de la vie de Fama, personnage principal du roman, que la voix narrative nous donne un aperçu de la corrélation entre la traduction et cette modernité romanesque :

«Fama bouillait de remords pour avoir tant combattu et détesté les Français un peu comme la petite herbe qui a grogné parce que le fromager absorbait tout le soleil ; le fromager abattu, elle a reç tout son soleil mais aussi le grand vent qui l'a cassée. (Soleils 22 ; nous soulignons)

...un petit garnement européen....remuant et impoli comme la barbiche d'un bouc... (Soleils 23 ; nous soulignons)

Fama demeura analphabète comme la queue d'un âne. (Soleils 24 ; nous soulignons)

Mariam gênait et elle était moqueuse comme une mouche, et, disait-on, féconde comme une souris. » (Soleils 152; nous soulignons)

Parlant des multiples concubinages de Fama dans la quête de cet héritier élusif, le narrateur se rabat encore sur le "comme": "Rien n'en sortit. Toutes [les femmes de Fama] cumulèrent des mois, parlèrent parfois de mariage, parcoururent des saisons, en abordèrent d'autres, mais toujours vides et sèches comme les épis de mil d'un bivernage écourté... " (Soleils 56 ; nous soulignons). Par ces récits de paroles et d'événements, le narrateur hétérodiégétique choisit comme objets de focalisation des acteurs ou personnages différents et dont les actions, attitudes ou états d'âme sont mis en relief d'une façon qui allie et fait équivaloir deux univers symboliques occupant des espaces différents dans l'échelle des valeurs socioculturelles. Le français est ainsi plus que jamais proche de l'imaginaire malinké. Véhicule de ce rapprochement sociolinguistique en butte contre toute hiérarchisation des langues et des cultures, le comparatif "comme" devient le signe primordial d'une réinvention linguistique et la matrice d'un entre-deux ou troisième espace qui transcende la dichotomie centre/périphérie. Ce vocable met fin à la violence épistémique du discours colonial. Il investit le texte d'« un code métissé du Soi et de l'Autre » (Bandia 128). L'usage du "comme" s'oppose aussi au " principe cognitif qui toujours incorpore et annule l'Autre dans le savoir du Même » (Caron 359). Il est opportun de noter que le rejet initial et la publication ultérieure de Les Soleils des indépendances par les Éditions du Seuil marquent respectivement l'aberrante politique de hiérarchisation des cultures qui place l'Autre en position d'infériorité et le triomphe contre ce postulat hégémonique. L'hybridation du récit a finalement droit à une certaine légitimité esthétique et peut ainsi se prévaloir d'un rôle didactique facilité par une traduction sur fond d'analogie et de modification sémantique ou translittération. Nous noterons que ce fait en soi est significatif puisqu'il permet à Kourouma d'actualiser, par l'écrit, les caractéristiques climatiques de l'espace malinké ou subsaharien pour instruire le narrataire non-malinké comme ce passage en témoigne: "Avez-vous déjà couché sur un tara? Il grince, geint comme si vous rouliez dans les feuilles mortes d'un sous-bois en plein harmattan » (Soleils 152; nous soulignons). Le narrateur avait déjà brossé des images spatio-temporelles familières pour un lectorat subsaharien ou malinké; nous relèverons celles du "fromager absorbait tout le soleil» et des « épis de mil d'un bivernage écourté ». Ces formulations relèvent de la "self-translation », une pratique par laquelle « [...] subaltern subjects seek to translate their knowledge into terms that make it apprehensible to distant metropolitan subjects » (Pratt 34).

Par ailleurs, l'interpellation d'un narrataire inaccoutumé au vécu réel ou imaginaire du Malinké, comme le besoin d'expliciter l'expérience de la tara en témoigne, souligne un autre trait de la structure du récit dans Les Soleils des indépendances, notamment l'interrogation formulée par le narrateur. À travers l'interrogation, l'exclamation, le recours à la répétition et à l'introspection 
narrativisée $e^{13}$, Kourouma s'adonne à une traduction romanesque des pratiques orales de la palabre comme il nous le révèle lui-même:

Q: « Vous utilisez également des techniques narratives différentes, néanmoins toutes empreintes de l'oralité pour rendre la matière de ces romans. Qu'est-ce qui vous a guidé vers ce choix? Serait-ce le cas de l'innovation qui appelle ou féconde l'innovation?

R: Non, ce n'est pas pour le fait de faire de l'innovation puisqu'il arrive que mon objectif est d'être authentique dans le sens africain. Par exemple, quand je parle des Soleils des Indépendances, je fais ce qui se fait comme palabres. Cela correspond à cela. Cela permet de traduire la situation qui était présente. » (Ouedraogo 774; nous soulignons)

La transcription des subtilités langagières de la palabre dans le récit consacre une façon culturelle de se dire et de se révéler au monde extérieur par le romanesque. C'est à cela que se résume d'ailleurs l'emploi du topos animal à l'instar du "comme" dans Les Soleils des indépendances.

La traduction n'est pas seulement du ressort de l'analogie interculturelle chez Kourouma. En tant que quête d'une forme d'équivalence (Pratt, Ibid. 33), elle rend parfois compte des valeurs intrinsèques d'une seule culture ou texte-source sans besoin de recourir à une comparaison interculturelle explicite. La traduction ou l'équivalence transcrite en français ne reproduit que les signifiés culturels de l'espace originel de l'auteur. Ainsi, Kourouma traduit ce jeu d'équivalence entre homme et animal par des métaphores tantôt positivées (le totem panthère), tantôt méprisées ou méprisables (la bâtardise). L'auteur les puise essentiellement de l’imaginaire socioculturel malinké. En effet, le paradigme analogique homme/animal se dit par des proverbes et des images métaphoriques endogènes, le but étant de mettre en relief la synonymie entre la bâtardise ou hybridation culturelle aberrante et la régression vers l'animalité, d'une part, et entre la déchéance sociale et la rapacité animalière, d'autre part. En bref, un bâtard culturel ou un malinké déchu équivaut à un animal et pas n'importe lequel, cependant. Ces quelques exemples suffiront à illustrer ce point:

«Lui, Fama, né dans l'or, le manger, l'honneur et les femmes! Éduqué pour préférer l'or à l'or, pour choisir le manger parmi d'autres, et coucher sa favorite parmi cent épouses! Qu'était-il devenu? Un charognard.» (nous soulignons)

«C'était une byène qui se pressait. » (Soleils 12) (nous soulignons)

«Il saura que l'byène a beau être édentée, sa bouche ne sera jamais un chemin de passage pour le cabrin.» (Soleils 17 ; nous soulignons)

«Impossible de dire un mot. Une meute de chiens en rut. tous ces assis de damnés de Malinkés se disant musulmans hurlèrent, se hérissèrent de crocs et d'injures. La limite était franchie. » (Soleils 18) (nous soulignons)

Par cette analogie à focalisation tantôt externe tantôt interne ${ }^{14}$, Kourouma accomplit deux tâches narratives. Il met en relief le parler malinké, d'une part, tout en caractérisant la pathologie culturelle qui règne dans cette société postcoloniale, d'autre part. Par celle-ci, l'auteur assimile l'espace des indépendances à une aire d'hybridation négative de l'homme qui devient l'équivalent de l'animal. Si

\footnotetext{
${ }^{13}$ En faisant la distinction entre le récit d'événements et le récit de paroles, Genette fait correspondre le discours narrativisé au récit d'événements et le discours rapporté au récit de paroles. Ainsi dit-il, «il [le discours narrativisé] est repris en main par le narrateur, intégré dans son discours. Le discours est devenu un événement comme un autre. Il est inséré dans le discours narratif et il ne se distingue, théoriquement, en rien du récit d'événements » (Bal, Ibid., 27).

14 « Si le narrateur "ne dit que ce que sait le personnage", le récit est à focalisation interne, que la focalisation soit fixe, variable ou multiple. Genette entend par le récit focalisé ce que Blin appellerait un récit à restriction de champ et Pouillon un récit à vision avec. Le troisième type est le récit à focalisation externe, où le narrateur "en dit moins que n'en sait le personnage", le dernier étant donc représenté de l'extérieur » (Bal, ibid. 23). Ces deux types de focalisation sont bien présents dans Les Soleils des indépendances, Fama en sait parfois autant ou moins que le narrateur qui laisse libre cours aux introspections du personnage principal qui se donne le récit de sa condition sociale et spirituelle.
} 
Kourouma dépeint l'impact des indépendances sur l'Afrique à travers une œuvre qui se construit esthétiquement par une déhiérarchisation linguistique et culturelle, Chamoiseau inscrit le récit de Solibo Magnifique dans un contexte de choc culturel et de diglossie. L'auteur martiniquais est tout aussi sensible à la traduction, et sa pratique actualise une esthétique du dit polyphonique proposée comme une alternative à la pensée de l’Un.

\section{La traduction, un outil de réfutation de la pensée de l’Un}

Solibo Magnifique dépeint un vécu diglossique dont l'une des composantes, le créole en l'occurrence, est vouée aux gémonies par certains et acclamés par d'autres. Chamoiseau y donne libre cours à une pratique de la traduction qui « [...] aide[..] à éclairer l'impact de la traduction sur [...] une culture linguistique métropolitaine qui apparaît homogénéisante » (Bandia 125). Le récit est énoncé par un narrateur homodiégétique majeur, Marqueur de paroles, et des narrateurs homodiégétiques "mineurs". Le particularisme dans la structure de cette dernière œuvre est inscrit dans la multiplicité des voix narratives s'exprimant à la première et/ou à la troisième personne. Ici, l'homodiégétique s'adapte bien à la polyphonie et à la traduction.

Le récit du roman est construit autour de la mort inattendue du conteur Solibo Magnifique et prend la forme d'un recueil kaléidoscopique de témoignages rapportés par un narrateur/personnage qui n'hésite point à passer la parole à ses pairs et par la plume d'un écrivain dont certains de ses personnages s'adonnent aussi à l'écrit. Le texte romanesque laisse figurer un récit tripartite: le procès-verbal introductif rédigé par l'officier de police judiciaire, Évariste Pilon; les dits de Solibo qui figurent en dernière partie; et le centre textuel où les témoignages et l'enquête sur la mort du conteur se font et se concluent. La structure du récit est constituée par: 1. un début textuel où un personnage associé à l'espace moderne s'exprime par le biais de l'écrit; 2 . une fin où un autre personnage appartenant au monde du dit créole célèbre la parole en s'en faisant le maittre à l'instar du griot africain; 3. un milieu où une variété de personnages laisse entendre leurs voix en l'absence du conteur. Sa structure semi-linéaire laisse entrevoir la proéminence de la parole et du groupe par le "nous". Le mistricrii et le patat'si célèbrent ce " nous cohérent ${ }^{15}$ dans chacune de ces trois étapes du récit. Faisant appel à Barthes qui conçoit la phrase comme «l'ébauche d'un petit récit »(4), nous remarquerons que le court procès-verbal figurant au début du roman comporte certains syntagmes ou « unités narratives » qui font l'esquisse du « nous » dans son rapport à la parole:

«Nous nous transportons immédiatement sur les lieux [...]

Constatons ce qui suit: $[\ldots]$

Saisissons le tambour $[\ldots]$

Ces opérations effectuées, faisons transporter le corps à la morgue de l'hôpital Clarac [...] Les lieux étant ouverts, faisons entourer l'arbre et ses abords de barrières Vauban [...] [nous soulignons] » (Solibo Magnifique 2021)

L'introduction de la partie qui suit le procès-verbal indique aussi l'importance du groupe et de la parole dans le récit à travers deux unités narratives:

«MES AMIS!

LE MAÎTRE DE LA PAROLE

PREND ICI LE VIRAGE DU DESTIN

ET NOUS PLONGE

DANS LA DÉVEINE... » (Solibo Magnifique 23 ; nous soulignons)

15 Selon Chamoiseau et Confiant, le E-kraa ou « nous cohérent » s'exprime à travers « le lieu de la veillée [qui] [...] est celui du partage de valeurs et des différences » (62). 
Le groupe identifié dans ce dernier passage par l'expression affective Mes amis se situe dans la savane, lieu d'expression d'une oralité créole guettée par l'oubli. Il diffère de celui du procès-verbal écrit qui relève du discours policier, de l'officialité administrative et de la francisation. Ces deux groupes se retrouvent cependant dans l'espace du commissariat pour des interrogatoires autour de la mort jugée suspecte du conteur Solibo Magnifique. C'est ce face-à-face entre les proches de Solibo et les enquêteurs qui sert de tremplin à l'inscription de l'écriture-traduction dans le corps du récit. Cette écriture-traduction s'assimile à l'écriture du registre en cela qu'elle est d'abord amorcée dans un esprit d'unilatéralisme et de fermeture à l'Autre. La poétique de la traduction dans Solibo Magnifique n'a pas manqué de susciter de l'intérêt chez les critiques. Liesbeth De Bleeker, par exemple, reconnaît la traduction pratiquée par Chamoiseau comme le "produit interculturel d'un texte-source créole vers un texte-cible français » (96). De Bleeker souligne aussi la récurrence de cette pratique chez les créolistes et pour l'auteur antillais en général. ${ }^{16}$ Notre analyse, à l'instar de celle de De Bleeker, corrobore cette importance surtout pour les auteurs de la Créolité. Celle que nous menons ici est particulière puisqu'elle montre la façon dont l'écriture-traduction déconstruit une posture contemporaine de déni associée au fait historique qu'est l'écriture du registre. Revenant à sa mise en œuvre dans Solibo Magnifique, nous noterons que la traduction se déploie parfois dans des circonstances de tension linguistique et de confusion discursive. A l'audition des témoins, l'officialité impose l'usage du français bien que le créole soit compris par tous:

«--Nom, prénom, surnom, âge, profession, domicile?

--Hein? [...]

--Quelle manière de te crier ta manman a donné à la mairie, traduit Bouaffesse.

--An pas save...

--Il dit qu'il ne sait pas, inspecteur...

--Merci, Brigadier, mais je comprends le créole.

--Je dis ça pour te rendre service! Tu es un inspecteur, tu dois pas fouiller dans ce patois de vagabonds...

--C'est une langue, Brigadier.

--Tu as vu ça où?

$--\ldots$

--Et si c'est une langue, pourquoi ta bouche roule toujours un petit francais builé?

Et pourquoi tu n'ecris pas ton procès-verbal avec? » (Solibo magnifique 133 ; nous soulignons)

La tension linguistique est non seulement patente chez le suspect, mais elle transparaît plus dans l'échange entre l'inspecteur Pilon et le brigadier Bouaffesse. Le traducteur fétichise le français, et ce faisant, prend une posture politique qui renvoie à la prédation inhérente à l'écriture du registre avec sa manie de précipiter le Divers dans la pensée de l'Un. ${ }^{17}$ L'extrait suivant illustre encore mieux ce refus de reconnaître le Divers créole dans son acception du nom:

--Nom, prénom, surnom, âge, profession, domicile?

-- Hein ?

--Dis comment on t'appelle, explique Bouaffesse.

--Bête-Longue.

--C'est votre surnom ? Bien. Nom et prénom maintenant.

-- Hein?

--Quelle manière de te crier ta manman a donné à la mairie, traduit

Bouaffesse.

\footnotetext{
16 « [L]'écrivain antillais et guyanais, en particulier celui de la zone franco-créolophone qui englobe la Martinique, la Guadeloupe, la Guyane française et Haïti, est un traducteur masqué ou, plus exactement, un traducteur inavoué. » (Confiant cité par De Bleeker 96).

${ }^{17}$ Caron applique ce concept glissantien au roman de Kourouma. Chamoiseau l'a aussi théorisé en rapport avec l'écriture du registre qui est ici l'équivalent du procès-verbal tout en renvoyant au discours de Bouaffesse pendant l'interrogatoire. Nous jugeons très opportune notre appréciation de ce concept dans le cadre de l'analyse de Solibo Magnifique. En effet, ce roman fait ressortir cet aspect du refus du Divers et sa déconstruction dans un élan créoliste.
} 
--An pas save... (Solibo magnifique, p. 133 ; nous soulignons)

Le projet de la Créolité étant la revendication d'une identité plurielle amalgamée et la légitimation de la culture créole, Chamoiseau se fait un point d'honneur de défendre cet héritage. Il y réussit en usant d'une méthode de traduction qui non seulement hybride le texte au profit du divers, mais aussi sert de prétexte à une défense et illustration du créole comme langue. La réaction de Pilon qui jugea nécessaire de faire reconnaittre le statut de langue du créole à son subalterne, Brigadier Bouaffesse, est à situer dans ce mouvement de défense du créole. L'inspecteur adopte une posture élogieuse en faveur de cette langue en s'opposant à toute conception unilatérale de la culture. A l'instar de la traduction qui semble ne pas apprécier, il remet la langue et le vécu créoles dans leur droit. Par ailleurs, le rôle de traducteur et la façon de traduire de Bouaffesse prennent toute leur signification dans le cadre de cette réhabilitation. Le brigadier adopte la "voie du centre" puisqu'il conjugue le français local et le français de l'ailleurs pour parvenir à se faire comprendre. Cette négociation qui produit un mélange de traduction sourcière et cibliste ${ }^{18}$ est aussi en phase avec " l'actuelle culturelle », qui, selon Édouard Glissant, «[...] doit être ici menée par des Antillais, mais encore doit-elle être dirigée contre un système » (100).

Une des définitions de la traduction est qu'elle renvoie aux « [...] tranpositions where knowledges, practices, or symbols of one or institution get processed into the contents of another [...] With reconversion, the translation again produces something nonequivalent to the original, yet in some sense it reproduces the original »(Pratt 33-34). Ceci étant, le traducteur Bouaffesse part d'une posture initiale de rejet du créole par son association avec un autre imaginaire pour finalement se rabattre sur celui créole grâce à la traduction. L'enjeu étant en effet l'intercompréhension, Bouaffesse traduit la question française «Nom, prénom, surnom, âge, profession, domicile? » par une autre tout à fait créolisée: "Quelle manière de te crier ta manman a donné à la mairie [?] ». Il emploie ici ce que De Bleeker a identifié comme une des formes de traduction préconisées par les auteurs antillais, la « paraphrase » notamment (98).

L'intérêt de la traduction dans ce cas est qu'elle tient en effet d'une reconversion linguistique et culturelle qui rend compte de la perception créole du nom ${ }^{19}$ tout en illustrant la façon dont « [l]a Créolité, comme d'ailleurs d'autres entités culturelles, a marqué d'un sceau indélébile la langue française » (Éloge 47). Bouaffesse passe en effet du policier au sociologue doublé de linguiste par ses références aux réalités particulières qui sous-tendent l'identité individuelle du personnage créole. Le retour à l'imaginaire créole par le biais de la traduction laisse entrevoir le pouvoir de la mère sur l'identité de l'enfant. ${ }^{20}$ Ce retour permet aussi de relever la créolisation du nom. Le sujet créole ne dit pas “je m'appelle"; son nom traduit une manière d'être au monde que la mère donne par le "cri". Bouaffesse réhabilite ainsi et par inadvertance un pan de la culture et de l'être créoles dans leur

\footnotetext{
${ }^{18}$ Dans sa discussion des différents types de traduction, Bandia identifie une traduction cibliste qui est plus assimilatrice que la traduction sourcière qui elle a « pour mission d'accentuer les éléments provenant du texte source » (131-32). Par source, on peut lire le cadre socioculturel d'où les personnages sont originaires, et Bouaffesse traduit dans et en fonction de cet espace.

19 Voir à ce propos le cas de Gros-Lombric dans Chemin-d'école de Chamoiseau, Paris: Gallimard, 1994. Ici le rapport entre l'espace et le nom se trouve bien illustré. Ne sachant que son nom créole, Gros-Lombric dit au maitre n'avoir pas entendu son nom et le maître de le lui demander. A sa réponse, le maître réagit comme suit: «C’est ainsi, je présume, que l'on vous appelle à la maison et dans les bois avoisinants votre case ? » (52). Dans une interview qu'il a accordée à Maeve McCusker, Chamoiseau parle du caractère réel des noms qu’il introduit dans ses romans, « De la problématique du territoire à la problématique du lieu: un entretien avec Patrick Chamoiseau, » The French Review 73.4 (2000), 728-729.

${ }^{20}$ Glissant parle de la mère « [1'] unique catalyse de l'organisation et de la fonction familiale » dans Le discours antillais (89).
} 
complexité. La mère créole figure au cœur de cette réhabilitation qui est en soi un signe de transcendance inavoué de la pensée de l'Un. Par ailleurs, sa traduction produit aussi une langue à cheval entre le français et le créole. Elle participe donc à la figuration de la diversité de l'hybride dans le corps du texte. Celle-ci se prête à l'esthétique de l'hybridité comme un entre-deux sociolinguistique et s'oppose donc à la simplification inscrite dans l'écriture du registre. L'hybride ne se limite pas seulement à la reformulation de questions relatives à l'identité; cet entre-deux transparaît aussi dans la coexistence entre le nom, le prénom et le surnom français et créole. Quelques suspects sont identifiés comme suit dans la liste des témoins établie par les enquêteurs: " Pierre Philomène Soleil, crié Pipi », "Sosthène Versailles, crié Ti-Cal », "Charles Gros-Liberté, crié Charlot », «Édouard Zaboca, crié la Fièvre » (Solibo Magnifique 177, 178, 180).

Le dévoilement d'une langue par le truchement d'une autre apparaît aussi sous une forme de pratique normale d'intercompréhension sans aucune tension dans Solibo Magnifique. L'auteur associe cette traduction presque comique avec les proches du conteur créole bien avant l'arrivée de la police. C'est dans un contexte de silence inattendu, celui de Solibo, que la traduction intervient pour expliquer le diagnostic dramatique de l'arrêt brusque de la parole du conteur: « Au moment où l'on ne s'y attendait plus, Congo se redressa-ahuri: Iye fout! hanmay halansé tÿou hot la hou hay dégawé mèdsin, mi Ohibo la ha hay an honjesion anlê noula!...--Hein, que dis-tu?-Oh, Congo déraille: il prétend que Solibo est en train de mourir, qu'il lui faut un médecin... » (Solibo Magnifique 36). C'est grâce à ce diagnostic du vieux Créole en créole et sa traduction en français par un membre de l'assistance que la vérité du destin de Solibo se révèle. Cette dualité linguistique est un peu à l'image du nom du conteur. Solibo Magnifique porte un prénom qui renvoie à un terme employé dans le damier martiniquais (cf., Perret 194) et un nom insolite tiré du lexique français.

\section{Conclusion}

Les Soleils des indépendances renferme plusieurs traits narratologiques participant de l'esthétique et de la thématique de l'hybride chez Kourouma. Parmi ceux-ci, l'emploi du comparatif "comme" est d'une grande prépondérance puisqu'il exprime le mieux le contact linguistique et culturel qui déstabilise le fétichisme de la langue française. Ce substantif fait écho, dans son rôle analogique et de traducteur, aux concepts d'hybride romanesque et de transculturation. En rapprochant deux imaginaires sociolinguistiques, ce vocable célèbre la prise de parole de la périphérie contre toute hégémonie et purisme linguistique. L'usage de la métaphore fait aussi partie intégrante de ce processus d'autorévélation culturelle au monde. Ainsi, la culture malinké parvient finalement à s'affirmer dans la modernité du texte romanesque sous la plume d'un de ses propres membres et par le truchement de la langue française avec à l'appui une traduction en faveur de l'émergence d'un troisième espace discursif. Chamoiseau a quant à lui joué beaucoup plus sur une enquête policière pour créer une esthétique en phase avec le kaléidoscope ou la mosaïque culturelle créole dont se targuent les auteurs d'Éloge de la Créolité. Elle repose sur une pratique de la traduction qui nie d'abord le local dans l'esprit de la politique de francisation, pour ensuite le réinscrire sous une forme plus hybride du langage. Le refus du fétichisme linguistique et la légitimation de la créolité ont mené nos deux auteurs à inventer des récits dont l'esthétisation et le fond narratif transcendent l'unicité ou l'autarcie culturelle pour embrasser l'hétérogénéité et le dialogue interculturel. La redondance du pluriel dans le titre du roman de Kourouma, Les Soleils des indépendances, et l'association de deux imaginaires dans Solibo Magnifique, témoignent d'un paratexte à l'image de l'hybride multidimensionnel du contenu textuel. Le tout conjugué participe d'une configuration particulière de 
l'œuvre francophone qui exploite la traduction dans sa pratique d'une mimésis romanesque ouverte au renouveau esthétique et à l'expérimentation théorique. 


\section{Bibliographie}

Bâ, Amadou Hampâté. Oui mon commandant! Paris: Actes Sud, 1996.

Bal, Mieke. Narratologie: Essais sur la signification narrative de quatre romans modernes. Paris: Editions Klincksieck, 1977.

Bandia, Paul. Cheikh Anta Diop: Translation at the Service of History. In Agents of Translation, Paul Bandia et John Milton, eds. Amsterdam: John Benjamins Publishing Company, 2009. 209-227.

---. «Le concept bermanien de l'“Étranger” dans le prisme de la traduction postcoloniale. » TTR 14.2 (2001): 123-139.

Barnabé, Jean, Patrick Chamoiseau et Raphaël Confiant. Éloge de la Créolité. Paris : Gallimard, 1993.

Barthes, Roland. «Introduction à l'analyse structurale des récits. » Communications 6 (1966): 1-27.

Britton, Celia. Édouard Glissant and Postcolonial Theory: Strategies of Language and Resistance. Charlotteville: University of Virginia Press, 1999.

Caron, David. « Pour une poétique de l'opacité: Ahmadou Kourouma, Édouard Glissant et l'espace de la Relation francophone. » Revue canadienne de littérature comparée 25.3-4 (1998) : 348-362.

Chamoiseau, Patrick. Chemin d'école. Paris: Gallimard, 1994.

---. Solibo Magnifique. Paris: Gallimard, 1988.

Chamoiseau, Patrick et Raphaël Confiant. Lettres créoles. Tracées antillaises et continentales de la littérature 1635-1975. Paris: Hatier, 1991.

De Bleeker, Liesbeth. « Scénographie postcoloniale et surconscience traductive dans Solibo Magnifique de Patrick Chamoiseau. » In L'écrivain caribéen, guerrier de l'imaginaire. Kathleen Gyssels \& B. Ledent, eds. Amsterdam: Rodopi, 2008. 83-106.

Gassama, Makhily. La Langue d'Ahmadou Kourouma ou le français sous le soleil d'Afrique. Paris: Karthala, 1995.

Glissant, Édouard. Le discours antillais. Paris: Seuil, 1981.

Gyasi, Kwaku A. «The African Writer as Translator: Writing African Languages through French Author(s). » Journal of African Cultural Studies 16.2 (2003): 143-159.

Koné, Amadou. «Discourse in Kourouma's novels: Writing Two Languages to Translate Two Languages. » Research in African literatures 38.2 (2007): 109-123.

Kourouma, Ahmadou. Les Soleils des indépendances. Paris: Seuil, 1970.

McCusker, Maeve. «De la problématique du territoire à la problématique du lieu : un entretien avec Patrick Chamoiseau. » The French Review 73.4 (2000): 728-729.

Moncef, S. B. «Ahmadou Kourouma, écrivain africain. » Afrique littéraire et artistique 10 (1970) : 2-8.

Murdoch, Adlai. Creole Identity in the French Caribbean Novel. Florida: University Press of Florida, 2001.

Ouédraogo, Jean. «Entretien avec Ahmadou Kourouma. » The French Review 74.4 (2001): $772-$ 785.

Parry, Benita. Postcolonial Studies: A Materialist Critique. London: Routledge, 2004.

Perret, Delphine. La Créolité, espace de création. Paris: Ibis Rouges Editions, 2001.

Prabbu, Anjali. Hybridity: Limits, Transformations, Prospects. New York: State University of New York, 2007. 
Pratt, Mary Louise. «The Traffic in Meaning: Translation, Contagion, Infiltration.» Profession (2002): 25-36.

Young, Robert. Colonial Desire: Hybridity in Theory, Culture and Race. London: Routledge, 1995. 